\title{
Phase separation and magnetic order in K-doped iron selenide superconductor
}

\author{
Wei Li ${ }^{1}$, Hao Ding ${ }^{1}$, Peng Deng ${ }^{1}$, Kai Chang${ }^{1}$, Canli Song ${ }^{1}, \mathrm{Ke} \mathrm{He}^{2}$, Lili Wang ${ }^{2}$, Xucun Ma², \\ Jiang-Ping $\mathrm{Hu}^{3}, \mathrm{Xi} \mathrm{Chen}^{1 \star}$ and Qi-Kun $\mathrm{Xue}^{1 \star}$
}

\begin{abstract}
The newly discovered alkali-doped iron selenide superconductors ${ }^{1,2}$ not only reach a superconducting transition temperature as high as $32 \mathrm{~K}$, but also exhibit unique characteristics that are absent from other iron-based superconductors, such as antiferromagnetically ordered insulating phases $^{3,4}$, extremely high Néel transition temperatures ${ }^{5,6}$ and the presence of Fe vacancies and ordering ${ }^{7-10}$. These features have generated considerable excitement as well as confusion, regarding the delicate interplay between Fe vacancies, magnetism and superconductivity ${ }^{11-13}$. Here we report on molecular beam epitaxy growth of high-quality $\mathrm{K}_{x} \mathrm{Fe}_{2-y} \mathrm{Se}_{2}$ thin films and in situ low-temperature scanning tunnelling microscope measurement of their atomic and electronic structures. We demonstrate that a $\mathrm{K}_{x} \mathrm{Fe}_{2-y} \mathrm{Se}_{2}$ sample contains two distinct phases: an insulating phase with well-defined $\sqrt{ } 5 \times \sqrt{5}$ order of Fe vacancies, and a superconducting $\mathrm{KFe}_{2} \mathrm{Se}_{2}$ phase containing no Fe vacancies. An individual Fe vacancy can locally destroy superconductivity in a similar way to a magnetic impurity in conventional superconductors. Measurement of the magnetic-field dependence of the Fe-vacancy-induced bound states reveals a magnetically related bipartite order in the tetragonal iron lattice. These findings elucidate the existing controversies on this new superconductor and provide atomistic information on the interplay between magnetism and superconductivity in iron-based superconductors.
\end{abstract}

Two central issues in studying quantum materials are the quality of samples and sensitivity of experimental probes. Molecular beam epitaxy (MBE) is one of the most powerful techniques for growth of thin films with precisely controlled composition and crystalline quality. The combination of MBE with a low-temperature scanning tunnelling microscope (STM) in the same ultrahigh-vacuum (UHV) environment avoids the complexity due to various irregularities in materials and ambient contamination, and enables us to reveal the intrinsic properties ${ }^{14}$. Here we extend the unique capability of the combined system to study ternary iron selenide.

Our experiments were conducted in a Unisoku UHV lowtemperature (down to $0.4 \mathrm{~K}$ ) STM equipped with an MBE chamber for thin-film growth. Magnetic field up to $11 \mathrm{~T}$ can be applied perpendicular to the sample surface. We grew $\mathrm{K}_{x} \mathrm{Fe}_{2-y} \mathrm{Se}_{2}$ on graphitized $6 \mathrm{H}-\mathrm{SiC}(0001)$ substrate, which follows the island growth mode. The size of an island is typically $100 \mathrm{~nm} \times 100 \mathrm{~nm}$. Two distinct regions (marked by I and II in Fig. 1b), coexisting side by side, are clearly revealed on each island, indicating that phase separation ${ }^{7,13}$ occurs.

The STM image with atomic resolution (Fig. 1c) of region I exhibits a centred rectangular lattice structure. The periods along the two orthogonal directions are 5.5 and $14.1 \AA$, respectively, as shown in Fig. 1c. Comparison with X-ray diffraction data suggests that the orientation of the film is (110) (see Fig. 1d) instead of the natural cleavage plane (001). Thus the STM actually images the cross-section of the layered material. The (110) plane is terminated with $\mathrm{K}$ and Se atoms. The $\mathrm{K}$ atoms are visible at positive bias and form atomic rows (Fig. 1c), which are $7.05 \AA$ apart and oriented along the [110] direction. At negative bias, the Se atomic rows (indicated by black dots in Fig. 1e) appear and zigzag through the $\mathrm{K}$ atoms. These images are fully consistent with the atomic structure of the (110) surface (Fig. 1d). Furthermore, our STM observation shows that there are very few defects in region I. We therefore identify region I as the stoichiometric $\mathrm{KFe}_{2} \mathrm{Se}_{2}$.

Scanning tunnelling spectroscopy (STS) probes the quasiparticle density of states. In Fig. 1f, we show the STS at $0.4 \mathrm{~K}$ in region I. The spectrum exhibits a superconducting gap centred at the Fermi energy and two characteristic coherence peaks, indicating that the stoichiometric phase $\mathrm{KFe}_{2} \mathrm{Se}_{2}$ is a superconductor. As expected, the gap and coherence peaks gradually disappear at higher temperatures as shown in the temperature-dependent tunnelling spectra (Fig. 1g).

The STS reveals a double-gap structure. The larger gap $\Delta_{1}=4 \mathrm{meV}$ is half of the energy between the two pronounced coherence peaks. The smaller one, $\Delta_{2} \sim 1 \mathrm{meV}$, is estimated by the two shoulders near the Fermi energy in the spectra. The coherence peaks of the smaller gap are hardly distinguishable because of thermal broadening. A nearly isotropic gap has also been observed by angle-resolved photoemission spectroscopy (ARPES; refs 15-18) on the electron-like Fermi surfaces near $M$ and $\Gamma$ points. However, the gap measured by ARPES is more than twice as large as $\Delta_{1}$. The discrepancy may be related to the averaging over different regions. The gap $\Delta_{2}$ is too small to be resolved by ARPES.

The finite-size effect on superconductivity is negligible because the typical size of region I, $20 \mathrm{~nm}$ thick (along the [110] direction in $a-b$ plane) and $50 \mathrm{~nm}$ wide (along the $c$ and [110] directions), is much larger than the superconducting coherence length of $\mathrm{KFe}_{2} \mathrm{Se}_{2}$. Here the anisotropic coherence lengths $\xi_{a b} \sim 2 \mathrm{~nm}$ and $\xi_{c} \sim 0.5 \mathrm{~nm}$ can be estimated by the upper critical fields ${ }^{19,20}$ in the $a-b$ plane and along the $c$ direction using $H_{c 2}{ }^{a b}=\Phi_{0} / 2 \pi \xi_{a b} \xi_{c}$ and $H_{c 2}{ }^{c}=\Phi_{0} / 2 \pi \xi_{a b}{ }^{2}$, respectively, where $\Phi_{0}$ is the quantum of flux. On the other hand, the penetration depth ${ }^{21}$ of the material is much larger than the sample size. Under this circumstance, the magnetic field is essentially uniform in a sample. In contrast to our previous work on FeSe (ref. 14), vortex structure is not formed in $\mathrm{KFe}_{2} \mathrm{Se}_{2}$ owing to the large penetration depth when compared with the sample size. However, the effect of magnetic field on superconductivity in $\mathrm{KFe}_{2} \mathrm{Se}_{2}$ is still clearly manifested in STS.

\footnotetext{
${ }^{1}$ State Key Laboratory of Low-Dimensional Quantum Physics, Department of Physics, Tsinghua University, Beijing 100084, China, ${ }^{2}$ Institute of Physics, Chinese Academy of Sciences, Beijing 100190, China, ${ }^{3}$ Department of Physics, Purdue University, West Lafayette, Indiana 47907, USA. *e-mail:xc@mail.tsinghua.edu.cn; qkxue@mail.tsinghua.edu.cn.
} 
a

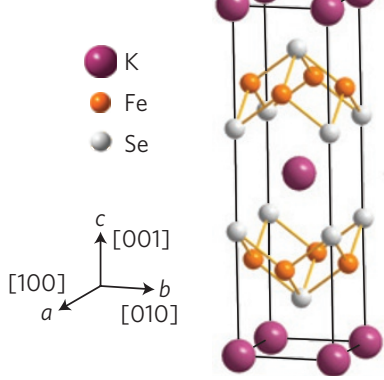

d
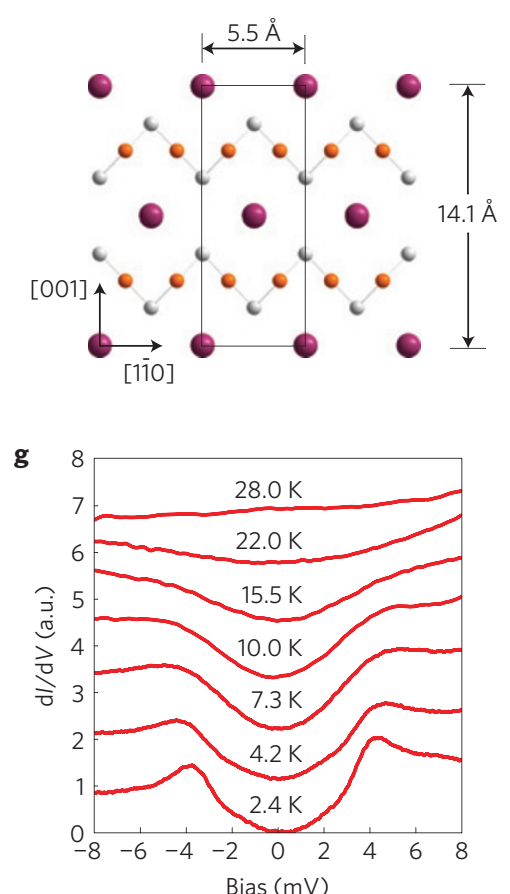

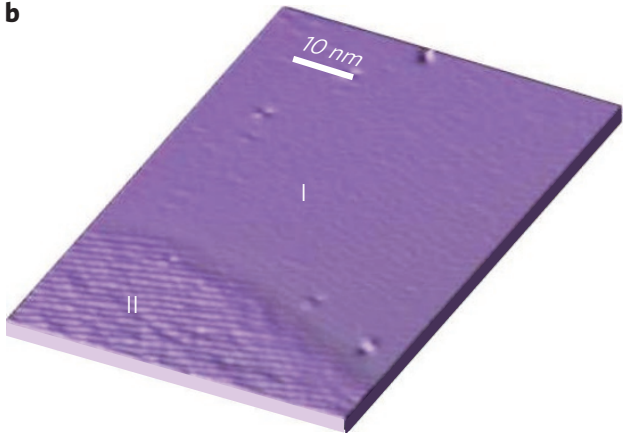

e

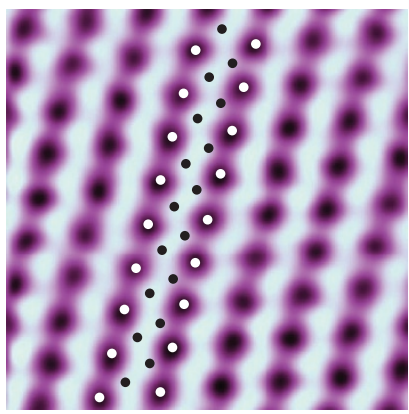

h

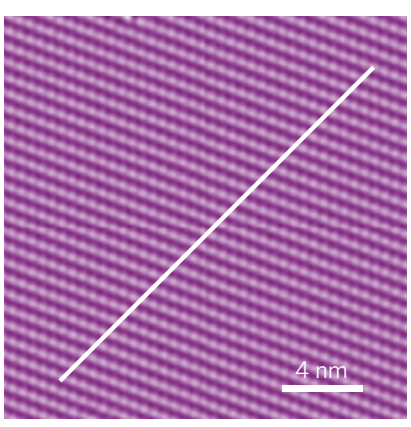

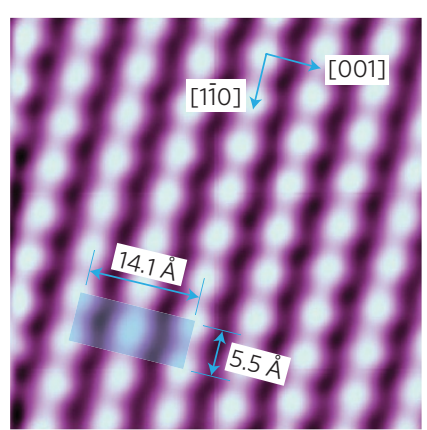

f
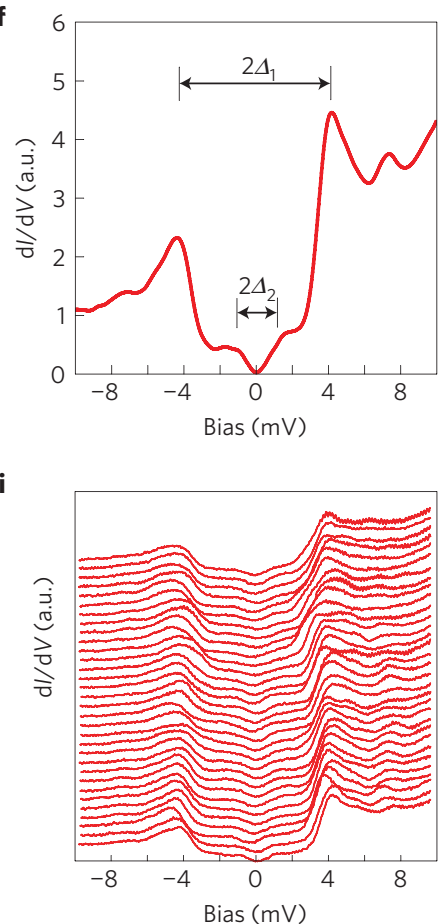

Figure 1 | MBE film and the superconducting phase of $\mathrm{K}_{\mathbf{x}} \mathrm{Fe}_{2-y} \mathrm{Se}_{2}$. $\mathbf{a}$, The crystal structure of $\mathrm{KFe}_{2} \mathrm{Se}_{2}$. The same conventions for atoms and Miller indices are used throughout. b, STM topographic image (bias voltage $V=3.9 \mathrm{~V}$, tunnelling current $I_{\mathrm{t}}=0.02 \mathrm{nA}$ ) of a $\mathrm{K}_{x} \mathrm{Fe}_{2-y} \mathrm{Se}_{2}$ film. The grain size is usually larger than $50 \mathrm{~nm} \times 50 \mathrm{~nm}$. Two distinct regions are labelled by I and II, respectively. c, Atomic-resolution STM topography of region I ( $5 \mathrm{~nm} \times 5 \mathrm{~nm}$, $V=0.15 \mathrm{~V}, l_{\mathrm{t}}=0.03 \mathrm{nA}$ ). The $\mathrm{K}$ atoms are visible at positive bias. $\mathbf{d}$. Atomic structure of (110) plane. $\mathrm{K}$ and Se atoms are in the topmost layer. Fe atoms are in the second layer. e, STM image of the same area as $\mathbf{c}$, but at negative bias. $V=-2 \mathrm{~V}, l_{\mathrm{t}}=-0.03 \mathrm{nA}$. Se atoms (marked by black dots) are visible. The positions of $\mathrm{K}$ atoms are marked by white dots. $\mathbf{f}$, Differential conductance spectrum in region I measured at $0.4 \mathrm{~K}$ ( set point, $V=14 \mathrm{mV}, l_{\mathrm{t}}=0.1 \mathrm{nA}$ ). $\mathbf{g}$, Temperature dependence of differential tunnelling conductance of the superconducting phase. Set point, $V=25 \mathrm{mV}, I_{t}=0.16 \mathrm{nA}$. The curves are offset vertically for clarity. $\mathbf{h}, \mathbf{i}$, Uniformity of the superconducting gap. The spectra in $\mathbf{i}$ were measured along the white line in $\mathbf{h}$. The size of the image in $\mathbf{h}$ is $20 \mathrm{~nm} \times 20 \mathrm{~nm}$. Set point, $V=14 \mathrm{mV}, \mathrm{I}_{\mathrm{t}}=0.1 \mathrm{nA}$.

A magnetic field breaks the time-reversal symmetry and weakens the superconductivity, giving rise to a reduction in the height of the coherence peak in the density of states (see Supplementary Fig. S1).

Because of the good sample quality, the superconducting gap exhibits high spatial homogeneity, as shown in Fig. 1h,i. Previous studies of iron-based superconductors usually suffer from various imperfections in the materials ${ }^{22}$. It is therefore crucial to prepare high-quality samples. As mentioned above, we solved this problem by MBE growth under well-controlled conditions (see also ref. 14). Furthermore, surface contamination is avoided because our film growth and STM study were conducted in a single UHV system. More significantly, by growing films with a (110)-oriented crystallographic surface where both $\mathrm{K}$ and Se atoms are exposed, we have realized the cross-sectional tunnelling configuration. Such a configuration for unconventional superconductors has been highly desirable, but very challenging to achieve owing to the difficulty in preparing a suitable surface for STM studies ${ }^{23}$.

Now we turn to region II, which shows a periodic stripe pattern (Fig. 1b). The STS in Fig. 2a exhibits an energy gap up to $0.43 \mathrm{eV}$ across the Fermi level, suggesting that this region is insulating. As well as the $\mathrm{K}$ atomic rows in the topmost layer, there is a superposed striped structure with a period of $14.0 \AA$ in the STM image (Fig. 2b). The stripes are along the $c$ axis and perpendicular to the $\mathrm{K}$ atomic rows. We attribute this superstructure to the $\sqrt{ } 5 \times \sqrt{ } 5$ pattern of Fe vacancies in the second atomic layer. They are visible in STM images because the electronic structure of the topmost layer is perturbed by the missing $\mathrm{Fe}-\mathrm{Se}$ bonds. Neutron scattering ${ }^{5}$, transmission electron microscopy ${ }^{7}$ and X-ray diffraction ${ }^{8}$ measurements have all revealed a $\sqrt{ } 5 \times \sqrt{ } 5$ order formed by Fe vacancies. Such a blocked checkerboard pattern ${ }^{9}$ gives rise to a $5 a_{\mathrm{Fe}} \sim 14.0 \AA$ periodicity in the (110) plane (Fig. 2c) and is consistent with the superstructure 

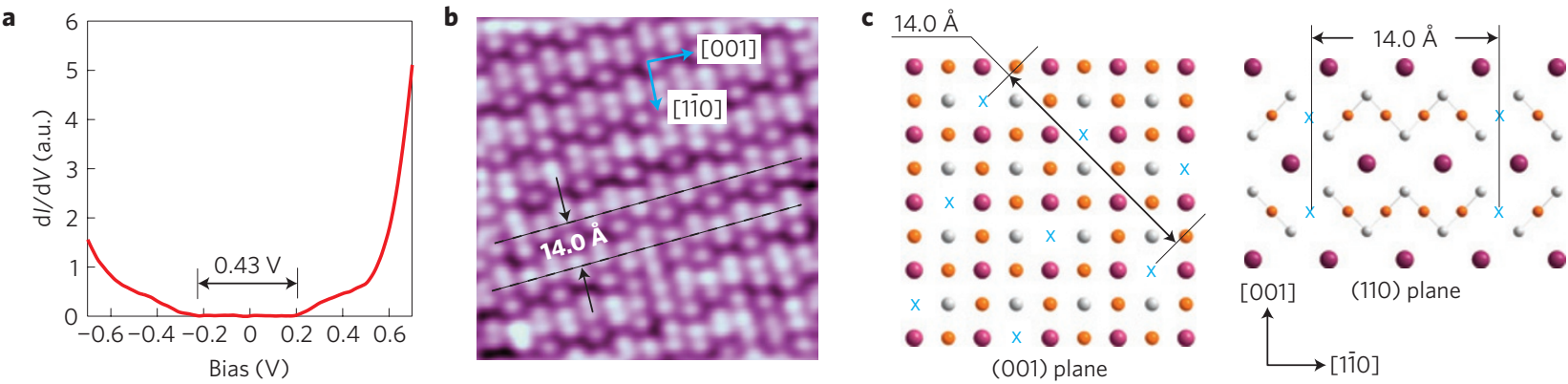

Figure 2 | The insulating phase. a, Differential conductance spectrum in region II (set point, $V=1.1 \mathrm{~V}, l_{\mathrm{t}}=0.26 \mathrm{nA}$ ). b, Atomic-resolution STM topography of region II $\left(10 \mathrm{~nm} \times 10 \mathrm{~nm}, V=2.9 \mathrm{~V}, I_{\mathrm{t}}=0.02 \mathrm{nA}\right)$. c, The structure of the $\sqrt{ } 5 \times \sqrt{ } 5$ Fe vacancy pattern as seen from (001) and (110) planes, respectively. The positions of Fe vacancies are marked by crosses.

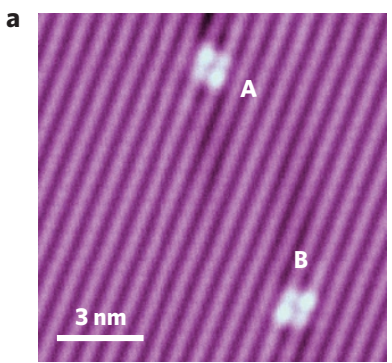

e

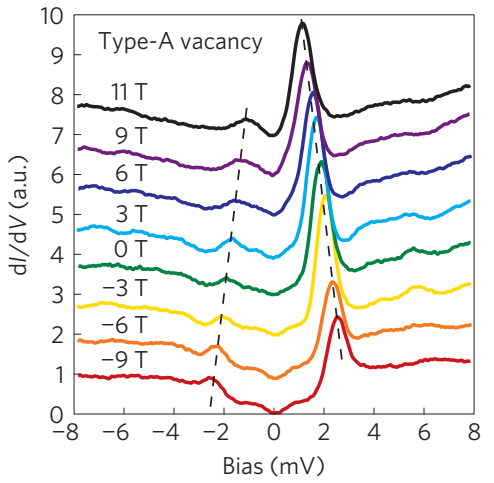

b
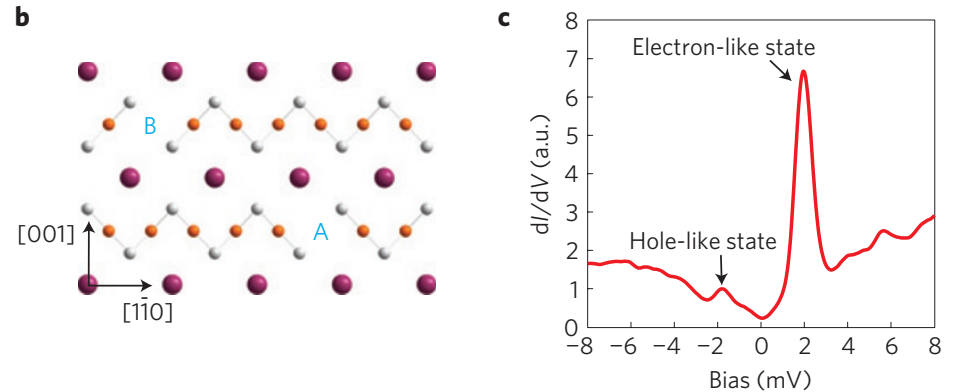

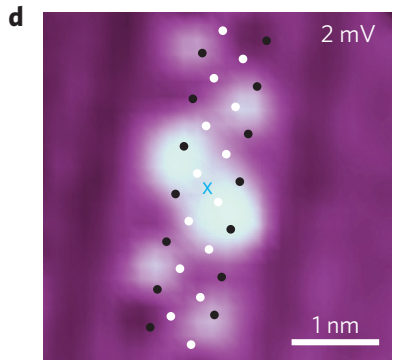

f

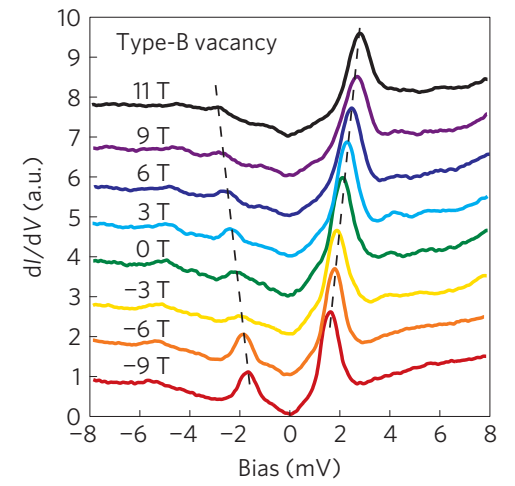

g

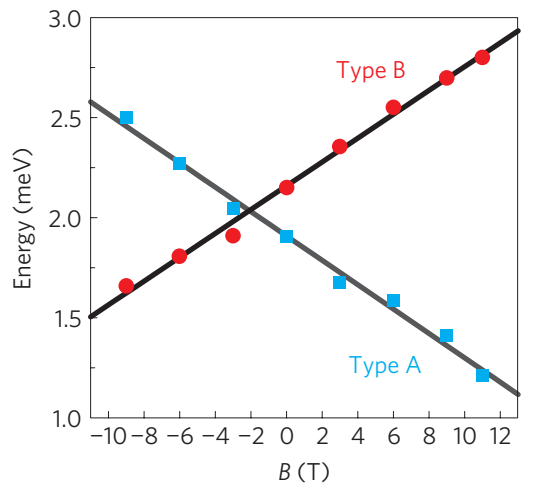

Figure 3 | Fe-vacancy-induced bound states in the superconducting gap of $\mathbf{K F e}_{2} \mathbf{S e}_{2} \cdot \mathbf{a}, \mathbf{b}, \mathrm{STM}$ topography $\left(12 \mathrm{~nm} \times 12 \mathrm{~nm}, \mathrm{~V}=30 \mathrm{mV}, l_{\mathrm{t}}=0.03 \mathrm{nA}\right)$ and atomic structure of Fe vacancies. Two types of vacancy are labelled by $A$ and $B$, respectively. $\mathbf{c}, \mathbf{d}, \mathrm{d} / / \mathrm{d} V$ spectrum measured at $0.4 \mathrm{~K}$ (set point, $V=15 \mathrm{mV}$, $\left.I_{t}=0.1 \mathrm{nA}\right)$ and density-of-states map of the bound state on a single Fe vacancy $(4 \mathrm{~nm} \times 4 \mathrm{~nm}, 2 \mathrm{mV})$. The tunnelling junction was set by $25 \mathrm{mV}$ and 0.16 $\mathrm{nA}$ during mapping. The positions of the Fe vacancy, $\mathrm{K}$ atoms and Se atoms are marked by a cross, black dots and white dots, respectively.

e-g, Magnetic-field dependence of the bound-state energies.

observed in our STM images. Here $a_{\mathrm{Fe}}$ is the in-plane distance between two neighbouring Fe atoms. The $\sqrt{ } 5 \times \sqrt{ } 5$ vacancy order leads to a composition of $\mathrm{K}_{x} \mathrm{Fe}_{1.6} \mathrm{Se}_{2}$, where $x$ is either 1 or 0.8 . As illustrated in Supplementary Fig. S2, among every five consecutive bright spots along the $\mathrm{K}$ atomic row, the one in the middle seems different from the other four. The STM image alone cannot show if this difference is due to the missing of one $\mathrm{K}$ atom $(x=0.8)$ or simply an electronic feature $(x=1)$, thus leaving the value of $x$ undetermined.

The above STM study, which combines the capabilities of imaging and spectroscopy together, has thus explicitly proved that $\mathrm{K}_{x} \mathrm{Fe}_{2-y} \mathrm{Se}_{2}$ is composed of two different phases, that is, superconducting $\mathrm{KFe}_{2} \mathrm{Se}_{2}$ and insulating $\mathrm{K}_{x} \mathrm{Fe}_{1.6} \mathrm{Se}_{2}$. The $\sqrt{ } 5 \times \sqrt{ } 5$ ordered pattern of Fe vacancies only exists in the insulating phase. As discussed below, our further experiment demonstrates that the superconducting phase has a hidden order related to Fe as well.

Defect-induced subgap states in superconductors, which can be detected by local probes such as STM/STS (refs 24-26), have often been used to uncover the nature of the superconducting state and magnetic interaction. To reveal the hidden order, we introduced defects (bright parallelograms in Fig. 3a) into the superconducting $\mathrm{KFe}_{2} \mathrm{Se}_{2}$ phase by annealing the sample at $450{ }^{\circ} \mathrm{C}$ for several hours. The atomically resolved STM images indicate that the topmost layer remains a perfect lattice without $\mathrm{K}$ or Se vacancies (Supplementary Fig. S3). The resulting defects are always located in the middle between two adjacent $\mathrm{K}$ atomic rows. We therefore attribute the parallelogram-shaped structures to single Fe vacancies in the second atomic layer (Fig. 3b). By examining the registration of $\mathrm{Fe}$ sites with respect to the Se lattice in the topmost layer, the $\mathrm{Fe}$ atoms in the (110) plane can be divided into two interpenetrating sublattices. The Fe vacancies on two different sublattices are labelled by $\mathrm{A}$ and $\mathrm{B}$, respectively. The atomic structure, chemical environment and STM images of these two types of vacancy are mirror images of each other.

An Fe vacancy carries spin and breaks superconducting pairing in the singlet channel through spin-flip scattering. The 


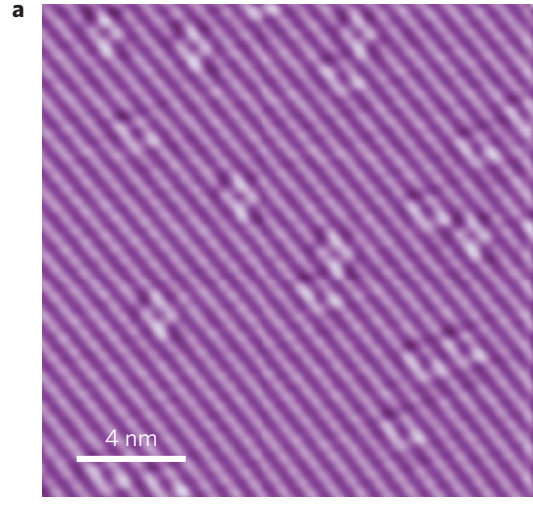

b

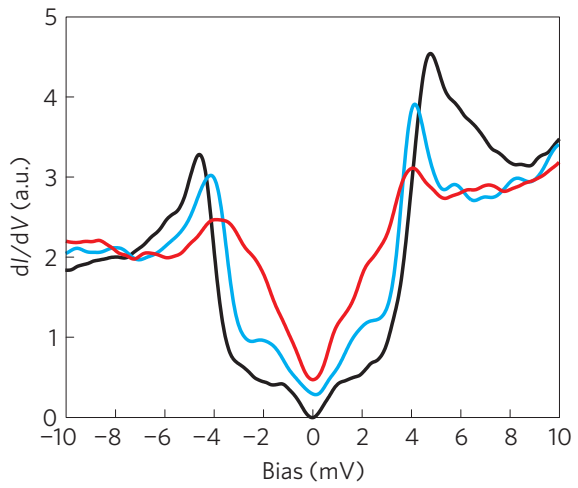

Figure 4 | Suppression of superconductivity of $\mathrm{KFe}_{2} \mathrm{Se}_{2}$ by Fe vacancies. a, STM topography $\left(18 \mathrm{~nm} \times 18 \mathrm{~nm}, V=60 \mathrm{mV}, I_{\mathrm{t}}=0.02 \mathrm{nA}\right)$ of an area with a high density of Fe vacancies. $\mathbf{b}$, Superconducting gaps (set point, $V=15 \mathrm{mV}, I_{\mathrm{t}}=0.1 \mathrm{nA}$ ) at various densities of vacancies. Black curve, no vacancies. Cyan curve, low density $\left(0.05 \mathrm{~nm}^{-2}\right)$ of vacancies. Red curve, high density $\left(0.1 \mathrm{~nm}^{-2}\right)$ of vacancies as shown in $\mathbf{a}$

exchange interaction $\boldsymbol{J} \mathbf{S} \cdot \boldsymbol{S}_{\mathrm{vac}}$, where $\mathbf{S}$ and $\mathbf{S}_{\mathrm{vac}}$ are the spins of a quasiparticle and a vacancy, respectively, gives rise to the bound quasiparticle states. The STS on a vacancy (Fig. 3c) shows strongly suppressed coherence peaks and a pair of resonances inside the superconducting gap, that is, an electron-like bound state at $1.9 \mathrm{mV}$ and a hole-like bound state at $-1.9 \mathrm{mV}$. Although the energies of the electron-like and hole-like states are symmetric with respect to zero bias, their amplitudes are different as a result of on-site Coulomb interaction ${ }^{27}$. The spatial extent of a resonance peak as shown in the $\mathrm{d} I / \mathrm{d} V$ mapping (Fig. $3 \mathrm{~d}$ ) is comparable to the coherence lengths in [110] and [001] directions. A vacancy is considered to be isolated if no others exist within twice the coherence length. The spectra on all isolated vacancies are identical, indicating that type-A and -B vacancies have the same magnitude of magnetic moment and the same exchange interaction $J$. The spectrum itself does not provide direct information on the orientation of magnetic moment.

To reveal the spin orientations of the two types (A and B) of vacancy, we apply a magnetic field perpendicular to the sample surface to break the rotational symmetry. So far, the magnetic-field effect on defect-induced subgap resonance has not been observed. However, in $\mathrm{KFe}_{2} \mathrm{Se}_{2}$, the observation of such an effect becomes possible owing to the high upper critical field and large penetration depth. As clearly shown in Fig. 3e,f, the energy of the subgap resonance in $\mathrm{KFe}_{2} \mathrm{Se}_{2}$ exhibits a linear dependence on magnetic field $\mathbf{B}: E=E_{0}+g \mu_{\mathrm{B}} \mathbf{B} \cdot \mathbf{S}$, where $\mu_{\mathrm{B}}$ is the Bohr magneton and $g$ the Landé factor. Fitting the data to a line (Fig. $3 \mathrm{~g}$ ) gives $g=2.070 \pm 0.07$, close to 2.0023 of a free electron. The most striking behaviour of the field effect is that the peaks on type-A and B vacancies shift in opposite directions with magnetic field. The opposite shifting suggests that the two types of vacancy have different spin orientations, implying a magnetically related bipartite order in the tetragonal Fe lattice. The bipartite spin structure is barely influenced by the applied magnetic field because of the relatively strong antiferromagnetic exchange coupling between neighbouring spins. Such bipartite structure may account for the large electron-like Fermi surface sheet with weak intensity near the $\Gamma$ point in ARPES data ${ }^{17,18}$ through Brillouin-zone folding.

The interplay between magnetism and superconductivity is one of the most intriguing phenomena in high-critical-temperature $\left(T_{\mathrm{c}}\right)$ superconductors. The technique mentioned above can sensitively probe local magnetism in superconductors and may help to understand how superconductivity arises in high- $T_{\mathrm{c}}$ materials.

The role of $\mathrm{Fe}$ vacancies in the superconducting phase has been rather controversial in previous studies. For example, some experiments indicated that randomly distributed vacancies may help to stabilize the superconducting state ${ }^{13}$. To further elucidate the effect of Fe vacancies on superconductivity, we prepared samples with higher density of randomly distributed vacancies (see Fig. 4a) by UHV annealing. The STS in Fig. 4 b shows that a sample with high density of vacancies eventually becomes a gapless superconductor. Therefore, the Fe vacancies are always destructive to superconductivity in $\mathrm{KFe}_{2} \mathrm{Se}_{2}$.

\section{Methods}

The $\mathrm{K}_{x} \mathrm{Fe}_{2-y} \mathrm{Se}_{2}$ sample was grown on a graphitized $6 \mathrm{H}-\mathrm{SiC}(0001)$ substrate. High-purity $\mathrm{Fe}$ (99.995\%), Se (99.9999\%) and K were evaporated from two standard Knudsen cells and one alkali-metal dispenser (SAES Getters), respectively. The growth was carried out under Se-rich conditions with a nominal Se/Fe flux ratio of $\sim 20$, which is known to lead to stoichiometric and superconductive FeSe (ref. 14). To intercalate $\mathrm{K}$ atoms in between FeSe layers (see the schematic crystal structure of $\mathrm{KFe}_{2} \mathrm{Se}_{2}$ in Fig. 1a), the substrate was held at $440^{\circ} \mathrm{C}$ during growth and the sample was subsequently annealed at $470^{\circ} \mathrm{C}$ for three hours. The $\mathrm{K}$ flux is relatively flexible, which determines the area ratio of the two different phases.

Received 1 August 2011; accepted 27 October 2011; published online 27 November 2011

\section{References}

1. Guo, J. et al. Superconductivity in the iron selenide $\mathrm{K}_{x} \mathrm{Fe}_{2} \mathrm{Se}_{2}(0 \leq x \leq 1.0)$. Phys. Rev. B 82, 180520(R) (2010).

2. Wang, A. F. et al. Superconductivity at $32 \mathrm{~K}$ in single-crystalline $\mathrm{Rb}_{x} \mathrm{Fe}_{2-y} \mathrm{Se}_{2}$. Phys. Rev. B 83, 060512(R) (2011).

3. Fang, M-H. et al. Fe-based superconductivity with $T_{\mathrm{c}}=31 \mathrm{~K}$ bordering an antiferromagnetic insulator in $(\mathrm{Tl}, \mathrm{K}) \mathrm{Fe}_{x} \mathrm{Se}_{2}$. Europhys. Lett. 94, 27009 (2011).

4. Chen, Z. G. et al. Infrared spectrum and its implications for the electronic structure of the semiconducting iron selenide $\mathrm{K}_{0.83} \mathrm{Fe}_{1.53} \mathrm{Se}_{2}$. Phys. Rev. B 83, 220507(R) (2011).

5. Bao, W. et al. A novel large moment antiferromagnetic order in $\mathrm{K}_{0.8} \mathrm{Fe}_{1.6} \mathrm{Se}_{2}$ superconductor. Chin. Phys. Lett. 28, 086104 (2011).

6. Pomjakushin, V. Yu. et al. Iron-vacancy superstructure and possible room-temperature antiferromagnetic order in superconducting $\mathrm{Cs}_{y} \mathrm{Fe}_{2-x} \mathrm{Se}_{2}$. Phys. Rev. B 83, 144410 (2011).

7. Wang, Z. et al. Microstructure and ordering of iron vacancies in the superconductor system $\mathrm{K}_{y} \mathrm{Fe}_{x} \mathrm{Se}_{2}$ as seen via transmission electron microscopy. Phys. Rev. B 83, 140505(R) (2011).

8. Zavalij, P.et al. Structure of vacancy-ordered single-crystalline superconducting potassium iron selenide. Phys. Rev. B 83, 132509 (2011).

9. Yan, X-W., Gao, M., Lu, Z-Y. \& Xiang, T. Ternary iron selenide $\mathrm{K}_{0.8} \mathrm{Fe}_{1.6} \mathrm{Se}_{2}$ is an antiferromagnetic semiconductor. Phys. Rev. B 83, 233205 (2011).

10. Ricci, A. et al. Nanoscale phase separation in the iron chalcogenide superconductor $\mathrm{K}_{0.8} \mathrm{Fe}_{1.6} \mathrm{Se}_{2}$ as seen via scanning nanofocused $\mathrm{X}$-ray diffraction. Phys. Rev. B 84, 060511(R) (2011).

11. Shermadini, Z. et al. Coexistence of magnetism and superconductivity in the iron-based compound $\mathrm{Cs}_{0.8}\left(\mathrm{FeSe}_{0.98}\right)_{2}$. Phys. Rev. Lett. 106, 117602 (2011).

12. Bao, W. et al. Vacancy tuned magnetic high- $T_{\mathrm{c}}$ superconductor $\mathrm{K}_{x} \mathrm{Fe}_{2-x / 2} \mathrm{Se}_{2}$. Preprint at http://arxiv.org/abs/1102.3674 (2011).

13. Shen, B. et al. Intrinsic percolative superconductivity in $\mathrm{K}_{x} \mathrm{Fe}_{2-y} \mathrm{Se}_{2}$ single crystals. Europhys. Lett. 96, 37010 (2011).

14. Song, C-L. et al. Direct observation of nodes and twofold symmetry in FeSe superconductor. Science 332, 1410-1413 (2011).

15. Zhang, Y. et al. Nodeless superconducting gap in $\mathrm{A}_{x} \mathrm{Fe}_{2} \mathrm{Se}_{2}(\mathrm{~A}=\mathrm{K}, \mathrm{Cs})$ revealed by angle-resolved photoemission spectroscopy. Nature Mater. 10, 273-277 (2011). 
16. Qian, T. et al. Absence of a holelike Fermi surface for the iron-based $\mathrm{K}_{0.8} \mathrm{Fe}_{1.7} \mathrm{Se}_{2}$ superconductor revealed by angle-resolved photoemission spectroscopy. Phys. Rev. Lett. 106, 187001 (2011).

17. Zhao, L. et al. Common Fermi-surface topology and nodeless superconducting gap of $\mathrm{K}_{0.68} \mathrm{Fe}_{1.79} \mathrm{Se}_{2}$ and $\left(\mathrm{Tl}_{0.45} \mathrm{~K}_{0.34}\right) \mathrm{Fe}_{1.84} \mathrm{Se}_{2}$ superconductors revealed via angle-resolved photoemission. Phys. Rev. B 83, 140508(R) (2011).

18. Wang, X-P. et al. Strong nodeless pairing on separate electron Fermi surface sheets in (Tl, $\mathrm{K}) \mathrm{Fe}_{1.78} \mathrm{Se}_{2}$ probed by ARPES. Europhys. Lett. 93, 57001 (2011)

19. Mun, E. D. et al. Anisotropic $H_{\mathrm{c} 2}$ of $\mathrm{K}_{0.8} \mathrm{Fe}_{1.76} \mathrm{Se}_{2}$ determined up to $60 \mathrm{~T}$. Phys. Rev. B 83, 100514(R) (2011).

20. Gao, Z. et al. Upper fields and critical current density of $\mathrm{K}_{0.58} \mathrm{Fe}_{1.56} \mathrm{Se}_{2}$ single crystals grown by one step technique. Preprint at http://arxiv.org/abs/1103.2904 (2011).

21. Torchetti, D. A. et al. ${ }^{77} \mathrm{Se}$ NMR investigation of the $\mathrm{K}_{x} \mathrm{Fe}_{2-y} \mathrm{Se}_{2}$ high- $T_{\mathrm{c}}$ superconductor $\left(T_{\mathrm{c}}=33 \mathrm{~K}\right)$. Phys. Rev. B 83, 104508 (2011)

22. Johnston, D. C. The puzzle of high temperature superconductivity in layered iron pnictides and chalcogenides. Adv. Phys. 59, 803-1061 (2010).

23. Fischer, $\varnothing$. et al. Scanning tunneling spectroscopy of high-temperature superconductors. Rev. Mod. Phys. 79, 353-419 (2007).

24. Yazdani, A. et al. Probing the local effects of magnetic impurities on superconductivity. Science 275, 1767-1770 (1997).

25. Ji, S-H. et al. High-resolution scanning tunneling spectroscopy of magnetic impurity induced bound states in the superconducting gap of $\mathrm{Pb}$ thin films. Phys. Rev. Lett. 100, 226801 (2008).
26. Balatsky, A. V., Vekhter, I. \& Zhu, J-X. Impurity-induced states in conventional and unconventional superconductors. Rev. Mod. Phys. 78, 373-433 (2006).

27. Salkola, M. I., Balatsky, A. V. \& Schrieffer, J. R. Spectral properties of quasiparticle excitations induced by magnetic moments in superconductors. Phys. Rev. B 55, 12648-12661 (1997).

\section{Acknowledgements}

We thank Y. G. Zhao for discussions. The work was financially supported by the National Science Foundation and Ministry of Science and Technology of China.

\section{Author contributions}

W.L., H.D., P.D., K.C. and C.S. carried out the experiments; K.H., L.W., X.M. and J-P.H. analysed the data; X.C. and Q-K.X. designed and coordinated the experiments; X.C. wrote the paper. All authors discussed the results and commented on the manuscript.

\section{Additional information}

The authors declare no competing financial interests. Supplementary information accompanies this paper on www.nature.com/naturephysics. Reprints and permissions information is available online at http://www.nature.com/reprints. Correspondence and requests for materials should be addressed to X.C. or Q-K.X. 